\title{
A Set of Important Kinetic Properties of Crystals and their General Statistical Calculations
}

\author{
National University "Lviv Polytechnic", 12 Bandery st., Lviv, Ukraine, 79013, e-mail: jabudjak@ukr.net
}

In non-equilibrium thermodynamics, the generalized equations of electric conductivity and heat conductivity are well known. They describe the response of the conductive medium to the effect of the drift fields in it and the magnetic field. These equations include phenomenological tensors and coefficients that determine the whole set of important kinetic properties of conducting crystals. Therefore, in order to find out the nature of crystal properties, it is necessary to clarify the nature of the set of kinetic tensors and the coefficients included in the general equilibrium of electrical conductivity and thermal conductivity. In this article, we will calculate the whole complex of these important quantities for isotropic crystals by statistical physics methods for general conditions of observation. And show the effect of spatial quantization on kinetic properties in 2D and 1D crystals.

Keywords: Gibbs potential, entropy, electrical conductivity, thermal conductivity, algorithm, tensor.

Article acted received 02.04.2018; accepted for publication 15.09.2018.

\section{Kinetic properties of crystals}

In non-equilibrium thermodynamics, it is well known that when an electric field with a strength ${ }^{E}$ or a temperature gradient $\nabla \underset{r}{r} T$ is created in a conducting crystal (these perturbations in a crystal can exist simultaneously), and next place this crystal in a magnetic field with an induction vector $B$, then we can observe transfer processes of electricity and heat. They are described by the first and second laws of non-equilibrium thermodynamics $\frac{d U_{e}}{d t}=-\operatorname{div} \stackrel{r}{q}+\underset{r}{j} E$, I- (the first law of non-equilibrium thermodynamics), $\frac{d S_{e}}{d t}=\frac{1}{T}\left(\stackrel{\mathrm{r} r}{j E}-\frac{{ }^{\mathrm{q}} \nabla \underset{r}{\mathrm{r}} T}{T}\right)$, II- (the second law of nonequilibrium thermodynamics)

In these equations: ${ }_{j}^{\prime}, \stackrel{\prime}{q}$ these are the electric current density vector and the heat flow vector, and, respectively $U_{e}, S_{e}$ the internal energy and entropy of the system.

In statistical physics it is shown that with the increase of entropy in the thermodynamic system there are phenomena of heat and electricity transfer (electrical conductivity and heat conductivity), and, conversely, if there is a phenomenon of transfer of heat and electricity in the system, then its entropy is increasing.
In non-equilibrium thermodynamics it is shown that the phenomena of electrical conductivity and thermal conductivity of a crystal are respectively described by the following generalized equations of electric conductivity and heat conductivity:

$$
\begin{aligned}
& \left.\stackrel{\mathrm{j}}{j}=\left(\sigma_{i k}\left(\begin{array}{l}
1 \\
B
\end{array}\right)\right)\right)^{\prime}-\left(\beta_{i k}\left(\begin{array}{l}
1 \\
B
\end{array}\right)\right) \nabla \underset{r}{\mathrm{r}} T \\
& \stackrel{\mathrm{r}}{q}=\left(\gamma_{i k}\left(\begin{array}{l}
1 \\
B
\end{array}\right)\right)^{\prime} E-\left(h_{i k}\left(\begin{array}{l}
1 \\
B
\end{array}\right)\right) \nabla \underset{r}{\mathrm{r}} T
\end{aligned}
$$

These equations describe the response of the conductive medium to the action of the electric field, the temperature gradient and the magnetic field. The phenomenological constants included in these equations $\left(\sigma_{i k}(\dot{B})\right),\left(\beta_{i k}(\dot{B})\right),\left(\gamma_{i k}(\dot{B})\right),\left(h_{i k}(\dot{B})\right)$ are tensors of kinetic coefficients, which in a certain way characterize many of the physical properties of the conducting medium (crystal). They possess the following properties of the Onzager symmetry:

$$
\begin{aligned}
& \left(\sigma_{i k}\left(\begin{array}{l}
\text { ' } \\
B
\end{array}\right)\right)=\left(\sigma_{k i}(-B)\right) ;\left(\beta_{i k}\left(\begin{array}{l}
\text { I } \\
B
\end{array}\right)\right)=\left(\beta_{k i}(-B)\right) ; \\
& \left(\gamma_{i k}\left(\begin{array}{l}
\mathrm{B} \\
B
\end{array}\right)\right)=\mathrm{T}\left(\beta_{k i}(-\stackrel{\prime}{B})\right)
\end{aligned}
$$

With linear transformation, taking into account Onzager's symmetry properties (3) we can take equation (1) and (2) to the following form: 


$$
\begin{aligned}
& \left.\dot{E}^{\prime}=\left(\rho_{i k}\left(B^{\prime}\right)\right)^{\prime} j+R\left({ }^{\prime} B\right)\left[{ }^{\prime} \times{ }^{\prime}\right]+\left(\alpha_{i k}\left(B^{\prime}\right)\right) \nabla r_{r} T+N(B) \mid B^{\prime} \times \nabla \underset{r}{r} T\right]
\end{aligned}
$$

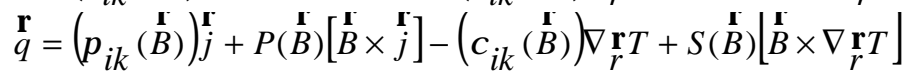

In these equations, square brackets indicate cross product of corresponding vectors.

The physical meaning of the phenomenological tensors and the coefficients of equations (4) - (5) can easily be determined by means of various phenomenological laws of non-equilibrium thermodynamics that describe the entire set of galvanomagnetic and thermomagnetic phenomena in semiconductor crystals.

Such an analysis shows that, in these equations, $\left(\rho_{i k}\right),\left(\alpha_{i k}\right),\left(\pi_{i k}\right),\left(\chi_{i k}\right)$ - are the material tensors of: the electrical resistivity, the effect of Zeebeck, the effect of the Peltier, and the heat conductivity of crystal; $R, N, P, S-$ this is the Hall effect coefficient, NernstEttinggshausen coefficient, Nernst and Rigi-Ledyuk coefficients respectively.

All tensors and coefficients, in accordance with the Onzager's principle of symmetry for kinetic coefficients, can be only even functions of magnetic induction vector.

These formulas make it possible to compare the results of experimental measurements with the conclusions of the macroscopic theory of nonequilibrium thermodynamics. They show that in the presence of a magnetic field in a crystal, usually simple phenomena of electrical conductivity and heat conductivity are complicating dramatically.

In this case, appear additional, so-called, galvanomagnetic and thermomagnetic effects. The first are due to the effect of the magnetic field on the ohmic part of the electric current, the second is on the thermal part. According to the general equation of conductivity (1), since the electric current consists of an ohmic part, which proportional electric field strength $\stackrel{\dot{E}}{E}$, and a thermal part, proportional to the temperature gradient $\nabla \mathrm{r} T$

All tensors and coefficients included in these equations have pragmatic values for modern solid-state electronics, because they characterize different properties of crystals used in the manufacturing of devices and systems of solid-state electronics.

\section{Statistical calculations of kinetic properties of crystals}

As shown in [1,2], all kinetic properties of a crystal are calculated by statistical methods with the help of a large canonical potential of Gibbs

$$
\Omega=-2 k T \underset{p}{\underset{p}{\ln } \ln }\left\{1+\exp \left(\frac{\mu+\Delta \mu \underset{p}{r}-\varepsilon \underset{p}{r}}{k T}\right)\right\},
$$

This potential is described in details in the quoted works, in which are given calculations of the whole set of important kinetic properties of isotropic semiconductor
3D, 2D and 1D crystals.

In modern literature, crystals with macroscopic dimensions are marked with 3D, thin-film crystals of microscopic thickness $d$ denoted by 2D mark, and threadlike crystals of thickness $d$ denote 1D signs.

The given calculations show that the kinetic properties of 3D crystals in a weak magnetic field. or in his absence, are described by the following algorithmic formulas:

$$
\begin{gathered}
\rho\left(\mu^{\bullet}, T\right)=\frac{1}{e n} \frac{J\left(0,0, \mu^{\bullet}, T\right)}{J\left(0,1, \mu^{\bullet}, T\right)}, \\
R\left(\mu^{\bullet}, T\right)=\frac{1}{z e n} \frac{J\left(0,0, \mu^{\bullet}, T\right) J\left(0,2, \mu^{\bullet}, T\right)}{J\left(0,1, \mu^{\bullet}, T\right)^{2}}, \\
\left.\alpha\left(\mu^{\bullet}, T\right)=\left(\frac{k}{z e}\right) \frac{J\left(1,1, \mu^{\bullet}, T\right)}{J\left(0,1, \mu^{\bullet}, T\right)}-\mu^{\bullet}\right], \\
N\left(\mu^{\bullet}, T\right)=\left(\frac{k}{e}\right) H_{\left.H^{(\mu}, T\right)}^{\bullet}\left[\frac{J\left(1,1, \mu^{\bullet}, T\right)}{J\left(0,1, \mu^{\bullet}, T\right)}-\frac{J\left(1,2, \mu^{\bullet}, T\right)}{J\left(0,2, \mu^{\bullet}, T\right)}\right], \\
\pi\left(\mu^{\bullet}, T\right)=T \alpha\left(\mu^{\bullet}, T\right),(11) \\
P\left(\mu^{\bullet}, T\right)=T N\left(\mu^{\bullet}, T\right) \\
\chi\left(\mu^{\bullet}, T\right)=\left(\frac{k}{e}\right)^{2} \frac{T}{\rho\left(\mu^{\bullet}, T\right)}\left[\frac{J\left(21, \mu^{\bullet}, T\right)}{J\left(0,1, \mu^{\bullet}, T\right)}-\left(\frac{J\left(1,1, \mu^{\bullet}, T\right)}{J\left(0,1, \mu^{\bullet}, T\right)}\right)\right], \\
U_{H}\left(\mu^{\bullet}, T\right)=\frac{J\left(0,2, \mu^{\bullet}, T\right)}{J\left(0,1, \mu^{\bullet}, T\right)} ; \\
U_{D}\left(\mu^{\bullet}, T\right)=\frac{J\left(0,1, \mu^{\bullet}, T\right)}{J\left(0,0, \mu^{\bullet}, T\right)}, \\
n\left(\mu^{\bullet}, T\right)=J\left(0,0, \mu^{\bullet}, T\right),
\end{gathered}
$$

These formulas show that the anisotropy caused by magnetic field disappears if the vector of magnetic induction $\stackrel{B}{B}$ of this field corresponds to the condition of a weak magnetic field $\left(\left(u(\varepsilon, T) B_{3}\right)^{2}<<1\right)$, or in case of its absence.

In these formulas $J\left(i, j, \mu{ }^{\bullet} T\right)$ is the main calculated functional. For an arbitrary isotropic law of dispersion of current carriers:

$$
\frac{p_{x}^{2}+p_{y}^{2}+p_{z}^{2}}{2 m m^{\bullet}}=\frac{p^{2}}{2 m m}=E(\varepsilon),
$$

this function has the following meaning: 


$$
\begin{gathered}
J\left(i, j, \mu^{\bullet}, T\right)=\int_{0}^{\infty}\left(\frac{\varepsilon}{k T}\right)^{i} u(\varepsilon, T)^{j} G(\varepsilon)\left(-\frac{d f_{0}}{d \varepsilon}\right) d \varepsilon=\int_{0}^{\infty} x^{i} u(x k T, T)^{j} G(x k T)\left(-\frac{d f_{0}}{d x}\right) d x= \\
=U(r, T)^{j} N_{C}(T) \int_{0}^{\infty} x^{i} u(x)^{j} G(x)\left(-\frac{d f_{0}}{d x}\right) d x=U(r, T)^{j} N_{C}(T) I\left(i, j, \mu{ }^{\bullet}, T\right) \\
J\left(0,0, \mu^{\bullet}, T\right)=\int_{0}^{\infty} G(\varepsilon)\left(-\frac{d f_{0}}{d \varepsilon}\right) d \varepsilon=\int_{0}^{\infty} g(\varepsilon) f_{0} d \varepsilon=n\left(\mu^{\bullet}, T\right), G(\varepsilon)=\int_{0}^{\varepsilon} g(\varepsilon) d \varepsilon,
\end{gathered}
$$

For an isotropic dispersion relation (16), which corresponds to the symmetry conditions of a crystalline lattice, a function $E(\varepsilon($ is a first degree homogeneous quantum-mechanical function of energy. For an isotropic parabolic dispersion relation, or a Kane dispersion relation, it has the following meanings, respectively:

$E(\varepsilon)=\varepsilon, \quad E(\varepsilon)=\varepsilon+\frac{\varepsilon^{2}}{E_{G}} \quad$. In addition, in this formula, $m$ - is mass of free electron, $m$-relative effective mass of current carrier in a crystal, $E_{G}-$ is band gap of the current carriers in the crystal.

For the given dispersion relation, the main calculated functional $J(i, j, \mu T)$ is described by formula (17), which uses the following notation: dimensionless functional

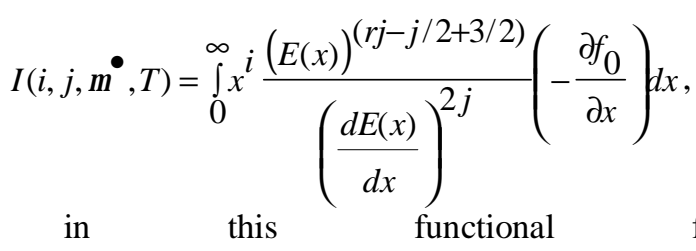

function $u(x k T, T)=U(r, T) \frac{E(x)^{(r-1 / 2)}}{\left(\frac{d E(x)}{d x}\right)^{2}}$ is called the scattering function. It describes the influence of scattering mechanisms of current carriers on defects of a crystalline lattice on the kinetic properties of crystals, and the scattering coefficient $r$ depends on the nature of the crystal and the nature of the defects; $U(r, T)$ - dimensional temperature function in current carriers mobility units, it is described by the following formula:

$$
U(r, T)=\left[\frac{1}{T}\left(U_{A} \delta(0, r)+U_{O} \delta(1, r)\right)+U_{I} \delta(2, r)\right]\left(m^{\bullet}\right)^{(r-5 / 2)} T^{(r-1 / 2)},
$$

where $U_{A}, U_{O}, U_{I}$ are the dimensional constants of the crystal, which depend on the nature of the crystal and the nature of the mechanisms of dispersion of current carriers on the defects of the crystal lattice, and $\delta(m, n)$ this is a known Kronecker delta, it has the following values: $\delta(m, n)=1$, if $m=n, \delta(m, n)=0$, if $m \neq n$;

$$
N_{C}(T)=\frac{8}{3 \sqrt{\pi}}\left(\frac{2 \pi m m^{\bullet} k T}{h^{2}}\right)^{3 / 2}
$$

The algorithmic formulas (7)-(15) with the functional (17) fully explain the nature of the whole set of actual kinetic properties of crystals. In this regard, they have practical applications in solid-state electronics research laboratories that synthesize various semiconductor crystals and experimentally examine their kinetic properties.

In this regard, let us consider the simple case: an isotropic crystal with parabolic dispersion relation for current carriers $\varepsilon=\frac{p^{2}}{2 m m^{\bullet}}$, and the dimensionless functional $I\left(l, j, \mu^{\bullet}, T\right)$ - by formula (18) for a parabolic dispersion relation is described by the following general formula:

$$
I\left(i, j, \mu^{\bullet}, T\right)=\int_{0}^{\infty} x^{[i+(r-1 / 2) j+3 / 2]}\left(-\frac{\partial f_{0}}{\partial x}\right) d x=F_{[i+(r-1 / 2) j+3 / 2]}\left(\mu^{\bullet}\right)
$$

\footnotetext{
In this formula, the function $F_{[i+(r-1 / 2) j+3 / 2]}\left(\mu^{\bullet}\right)$ with a lowercase index $[i+(r-1 / 2) j+3 / 2\rfloor-$ is well studied in the kinetic
}

theory of crystals, Fermi integral. Formula (21) makes it possible, with the help of Fermi integral, to calculate the entire set of kinetic properties (7) - (15) for crystals with an arbitrary level of degeneration of their current carriers. For non-degenerate charge carriers $\left(\mu^{\bullet}<-4\right)$, the 
Fermi integral has the following solution:

$$
\left.F[i+(r-1 / 2) j+3 / 2]]^{\bullet}\right)=\Gamma(i+(r-1 / 2) j+5 / 2) \cdot e^{\mu^{\bullet}}
$$

where $\Gamma(i+(r-1 / 2) j+5 / 2)$ - the gamma Euler's function.

So in this case we have:

$$
I\left(l, j, \mu^{\bullet}, T\right)=\Gamma(i+(r-1 / 2) j+5 / 2) \cdot e^{\mu^{\bullet}},
$$

In the case of strong degeneracy $\left(\mu^{\bullet}>+4\right)$, the Fermi integral (21) is well approximated by the following formula:

$$
F_{[i+(r-1 / 2) j+3 / 2]}\left(\mu^{\bullet}\right) \cong\left(\mu^{\bullet}\right)^{[i+(r-1 / 2) j+3 / 2]}
$$

therefore the dimensionless functional (18) is equal to:

$$
I(l, j, \mu) \cong \mu^{[i+(r-1 / 2) j+3 / 2]}
$$

Formulas (22) and (24) make it possible to calculate the kinetic properties of the crystal (7) - (15) with nondegenerate or strongly degenerate current carriers with a parabolic dispersion relation. According to these calculation algorithms, we have the following results of calculations:

a. for crystals with non-degenerate charge carriers:

$$
\begin{gathered}
\sigma\left(\mu^{\bullet}, T\right)=\operatorname{en}\left(\mu^{\bullet}, T\right) U_{D}(T), \\
R\left(\mu^{\bullet}, T\right)=\frac{1}{z e n\left(\mu^{\bullet}, T\right)} \frac{\Gamma\left(\frac{5}{2}\right) \Gamma\left(2 r+\frac{3}{2}\right)}{(\Gamma(r+2))^{2}}, \\
\alpha\left(\mu^{\bullet}, T\right)=\frac{k}{z e}\left[(r+2)-\mu^{\bullet}\right] \\
N\left(\mu^{\bullet}, T\right)=\frac{k}{e} U_{H}(T)\left(\frac{1}{2}-r\right),
\end{gathered}
$$

$$
\begin{gathered}
\pi\left(\mu^{\bullet}, T\right)=T \alpha\left(\mu^{\bullet}, T\right), \quad P\left(\mu^{\bullet}, T\right)=T N\left(\mu^{\bullet}, T\right), \\
\chi\left(\mu^{\bullet}, T\right)=\left(\frac{k}{e}\right)^{2} \sigma\left(\mu^{\bullet}, T\right) T(r+2) \\
U_{D}(T)=U_{0}^{(r)}(T) \frac{\Gamma(r+2)}{\Gamma\left(\frac{5}{2}\right)}, \\
U_{H}(T)=U_{D}(T) \frac{\Gamma\left(\frac{5}{2}\right) \Gamma\left(2 r+\frac{3}{2}\right)}{(\Gamma(r+2))^{2}}, \\
U_{H}(T)=U(r, T) \frac{\Gamma\left(2 r+\frac{3}{2}\right)}{(\Gamma(r+2))}, \\
n\left(\mu^{\bullet}, T\right)=2\left(\frac{\left.2 \pi m m{ }^{\bullet} k T\right)^{3 / 2}}{h^{2}} \cdot e^{\bullet}\right.
\end{gathered}
$$

b. for crystals with strongly degenerate charge carriers:

$$
\begin{gathered}
\sigma\left(\mu^{\bullet}, T\right)=e n(\mu) U_{D}(\mu, T) \\
R(\mu, T)=\frac{1}{z e n(\mu)}, \\
\alpha(\mu, T)=\frac{\pi^{2}}{3}\left(\frac{k}{z e}\right)\left(\frac{k T}{\mu}\right)(r+1) \\
N(\mu, T)=\frac{\pi^{2}}{3}\left(\frac{k}{e}\right) U_{H}(T)\left(\frac{k T}{\mu}\right)\left(\frac{1}{2}-r\right), \\
\pi(\mu, T)=T \alpha(\mu, T), \quad P(\mu, T)=T N(\mu, T), \\
\chi(\mu, T)=\frac{\pi^{2}}{3}\left(\frac{k}{e}\right)^{2} \sigma(\mu, T) T
\end{gathered}
$$

$$
\begin{gathered}
U_{H}(\mu, T)=U_{D}(\mu, T), \\
n(\mu, T)=\frac{8}{3 \sqrt{\pi}}\left(\frac{2 \pi m m^{\bullet} \mu}{h^{2}}\right)^{3 / 2},
\end{gathered}
$$

In the case of strong degeneration of current carriers, their drift mobility $U_{D}$ and Hall mobility $U_{H}$ are same, and the temperature function $U(r, T)$, for scattering current carriers on thermal fluctuations of the crystalline lattice, can be characterized by scattering indices $r=0$, or $r=1$ has such a value $U(r, T) \sim \frac{1}{T}$. This is a complex process of scattering.

For scattering of degenerated current carriers on an ionized dopant in a crystalline lattice, which is characterized by a scattering index $r=2$, both mobility $U_{H}(\mu, T)$ and $U_{D}(\mu, T)$ coincide. In this case
$U_{D}(\mu, T)=U_{H}(\mu, T)=U_{I}\left(m^{\bullet}\right)^{-\frac{1}{2}} \mu^{\frac{3}{2}}$ so the mobility of current carriers has no temperature dependence $U(r, T) \sim$ const.

In $2 \mathrm{D}$ and $1 \mathrm{D}$ crystals, spatial quantization of the energy spectrum for crystal current carriers is observed. That's why we can observe in such crystals, the correlation of their kinetic properties with thickness $d$. Indeed, in the cited literature it is shown that the main kinetic functional $J\left(i, j, d, \mu^{\bullet}, T\right)_{2 D} \quad$ and $J\left(i, j, d, \mu^{\bullet}, T\right)_{1 D}$ those that determine the kinetic properties of $2 \mathrm{D}$ and $1 \mathrm{D}$ crystals are described by the following algorithmic formulas:

$$
\begin{gathered}
J\left(i, j, d, \mu^{\bullet}, T\right)_{2 D}=J\left(i, j, \mu^{\bullet}, T\right) \exp \left(-F(d)_{2 D}\right), \\
J\left(i, j, d, \mu^{\bullet}, T\right)_{1 D}=J\left(i, j, \mu^{\bullet}, T\right) \exp \left(-F(d)_{1 D}\right),
\end{gathered}
$$

In these formulas, the correlation functions $F(d)_{1 D}$ 
and $F(d)_{2 D}$ has the following value:

$$
F(d)_{1 D}=\frac{3 \sqrt{\pi}}{8} \cdot \frac{F_{[i+(r-1 / 2) j+1]^{(\mu}}}{\left.\left.F_{[i+(r-1 / 2) j+3 / 2]}\right]^{(\mu}\right)} \cdot\left(\frac{\lambda_{D}(T)}{d}\right)\left(1-\frac{1}{\sqrt{\pi}} \cdot \frac{\left.F_{[i+(r-1 / 2) j+1 / 2}\right]^{\left(\mu^{\bullet}\right)}}{\left.F_{[i+(r-1 / 2) j+3 / 2}\right]^{\left(\mu^{\bullet}\right)}} \cdot\left(\frac{\lambda_{D}(T)}{d}\right)\right),
$$

$F(d)_{1 D}=\frac{3 \sqrt{\pi}}{8} \cdot \frac{F_{[i+(r-1 / 2) j+1]}\left(\mu^{\bullet}\right)}{F_{[i+(r-1 / 2) j+3 / 2]}\left(\mu^{\bullet}\right)}\left(\frac{\lambda_{D}(T)}{d}\right)$

and the function $\lambda_{D}(T)=\left(\frac{h^{2}}{2 \pi m m^{\circ} k T}\right)^{1 / 2}$ is the thermal de

Broglie wavelength; the functional $J\left(i, j, \mu^{\bullet}, T\right)$ is described by the formula (17).

Detailed method for analyzing experimental data

[1] J.S. Budjak, Gibbs Grand Thermodynamic Potential in the Theory of Kinetic Crystal Properties, Physics and chemistry of solid state, 18(1), 7-14 (2017); doi: 10.15330/pcss.18.1.7-14.

[2] J. Budjak, V. Chaban, Energetic and kinetic properties of semiconductor crystals, Lviv, ProstirM, 190 p. (2017). about some of the kinetic properties of 3D crystals using formulas (7)-(15), with Kane's dispersion relation, is given in the cited paper [2].

Budjak Ja.S. - Professor, Doctor of Physical and Mathematical Sciences.

\title{
Я.С. Буджак
}

\section{Множина важливих кінетичних властивостей кристалів та їх загальні статистичні розрахунки}

\author{
Національний університет „Львівська політехніка”, в. Бандери, 12, м. Львів, Украӥна, 79013, \\ e-mail: jabudjak@ukr.net
}

\begin{abstract}
В нерівноважній термодинаміці відомі узагальнені рівняння електропровідності та теплопровідності. Вони описують відгук провідного середовища на дію дрейфових полів в ньому та магнітного поля. В ці рівняння входять феноменологічні тензори та коефіцієнти, які визначають всю множну важливих кінетичних властивостей провідних кристалів. Отже, для вияснення природи властивостей кристала необхідно вияснити природу множини кінетичних тензорів і коефіцієнтів, які входять в узагальнені рівняння електропровідності та теплопровідності. В даній роботі вся множина цих важливих величин для ізотропних кристалів методами статистичної фізики розраховуються при загальних умовах спостереження, а також показано вплив просторового квантування на кінетичні властивості в $2 D$ та $1 D$ кристалах.
\end{abstract}

Ключові слова: потенціал Гіббса, ентропія, електропровідність , теплопровідність, алгоритм, тензор. 